Aus der chirurgischen Abteilung der Krankenanstalt Neumünster, Zürich (Chefarzt: Dr. Friedrich Brunner).

\title{
Über Störungen von seiten des Halssympathicus bei einfacher Struma und im Anschluß an deren operative Behandlung.
}

Von Dr. Werner Kaelin, Assistenzarzt.

Wiewohl in den letzten Jahrzehnten die Zahl der Arbeiten über die normale und pathologisch veränderte Schilddrüse zu einer kaum mehr zu sichtenden Fülle angewachsen ist, finden sich doch noch Erscheinungen, die wohl vereinzelt registriert, aber kaum je einmal im Zusammenhang gesichtet und beschrieben wurden, die aber manches Interessante sowohl vom theoretischen als rom praktisch-chirurgischen Standpunkte aus bieten: es sind das die Irscheinungen ron seiten des Halssympathicus bei einfacher Struma 1 ;.

Diese allein sollen hier gestüzt auf die Beobachtungen am Krankenmaterial der chirurgischen Abteilung der Krankenanstalt Neumünster in Zürich eine nähere Besprechung erfahren, während die Beziehungen des Sympathicus zum Morbus Basedowi und auch die Sympathicuslähmungen bei Struma maligna nicht in Bereich unserer Arbeit fallen. Denn daß Wachstum bei Struma maligna die benachbarten Halsorgane und damit auch cien Grenzstrang des Sympathicus relativ früh befällt, ist eine allgemein bekannte Erscheinung.

Hie: interessieren uns in erster Linie die Sympathicuserscheinungen, wie sie im Gefolge der einfachen benignen Struma auftreten, sodann jene, die im Anschluß an die Kropfoperation

I) So finden sich unter den in Hildebrandts Jahresberichten angeführten 2050 Arbeiten über Struma keine einschlägigen Arbeiten und auch dic schwcizerischen Kropfstatistiken versagen hicrin. 
zur Beobachtung gelangen. Der'Besprechung dieser beiden Kategorien sollen einige kurze anatomische und physiologische $\mathrm{Be}$ merkungen vorangehen.

Anatomie.

Die beiden Lappen der schon normalerweise in ihrer Form stark variierenden Glandula thyreoidea liegen dem seitlichen Umfang der oberen Trachealringe, der Cartilago cricoidea und thyreoidea an, reichen nach hinten bis zum Oesophagus und seitwärts außen bis an den Gefäßnervenstrang des Halses. (Arteria carotis communis medial, Vena jugularis lateral und Nervus vagus im hintern von diesen beiden gebildeten Winkel.)

Die einzelnen Gebilde dieses Stranges bleiben in ihren gegenseitigen topographischen Beziehungen unverändert vom Eintritt der Art. carotis in die Halsregion hinter der Articulatio sternoclavicularis bis zur Höhe der Cartilago thyreoidea. Sie sind von einer gemeinsamen Scheide eingeschlossen und durch lockeres Bindegewebe mit der Nachbarschaft fixiert.

Der Grenzstrang des Sympathicus liegt hinter der Vena jugularis interna, etwas außen vom Nervus vagus und der Arterie auf den Processus transversi der Cervikalwirbel, von denen ihn jedoch die Mm. longi colli et capitis, sowie die Fascia pracvertebralis trennen. Über sein Verhältnis zu dieser Fascie bestehen verschiedene Auffassungen, die wohl durch die große Variabilität der hier in Betracht kommenden Verhältnisse zu erklären sind. Jedenfalls ist der Grenzstrang auf der die oben genannten Muskeln bedeckenden Fascie festgeheftet. Nach Drobnik existiert ein den Nerven von den andern Gebilden des Halses deutlich abgrenzendes Fascienblatt (Sympathicus. scheide) in jedem Falle.

Durch diese anatomische Anordnung ist der Sympathicus vor Lageveränderungen geschützt, wie sie in charakteristischer Weise am Gefäßnervenstrang des Halses auftreten.

Auf der Höhe des 5. Halswirbels tritt der Grenzstrang in Beziehung zur Arteria thyreoidea inferior. Der gewöhnliche Verlauf dieser Arterie ist der: Nachdem sie aus dem Truncus thyreocervicalis entsprungen, steigt sie, den Mm. scaleni anliegend ziemlich senkrecht empor bis zur Höhe des 5. Cervikal- 
wirbels und wendet sich in einem nach vorn konkaven Bogen in schiefer Richtung zur Schilddrüse, wobei sie zwischen dem Gefäßnervenstrang vorn und der Arteria vertebralis hinten durchtritt.

Der Sympathicus selbst kann teils hinter der Art. thyr. inf., teils vor derselben liegen. Dieser letztere Befund soll der häufigere sein. Mitunter teilt sich der Grenzstrang oberhalb der Art. thyr. inf. in zwei Äste, die sich unterhalb derselben wieder vereinigen können, so daß also die Arterie durch einen Schlitz des Nerven hindurchtritt.

Bei Vorhandensein eines Ganglion cervicale medium, das eben hier an der Kreuzungsstelle liegt, kann es vorkommen, $\mathrm{da} B$ ein Teil des Knotens über, ein Teil unter der Arterie liegt; diese Ganglienteile sind dann durch zwei ziemlich starke Nervenästchen verbunden, zwischen denen die Arterie zum Durchtritt eben Platz findet. Bei der anatomischen Darstellung dieser Verhältnisse drängt sich die Frage auf, ob eine Erweiterung des Gefäßes bei Kropf ohne Beeinträchtigung der Funktion der Sympathicusfasern möglich sei. (D robnik.)

Der Sympathicus besorgt fast ausschlieBlich die Innervation der Schilddrüse (B riau) und bildet um die Arterien, hauptsächlich um die Art. thyr. inf. wahre Plexus, die wohl bei der Unterbindung derselben fast immer geschädigt oder zerstört werden, ohne daß besondere merkliche Erscheinungen eintreten.

Für die im Gebiete des Kropfes auftretenden Störungen kommen bei Verletzung des Nerven jene Fasern in Betracht, die aus dem 8. Cervikal- und I. Dorsalsegment des Rückenmarkes als Rami communicantes in das Ganglion cervicale inferius des Grenzstranges treten. Hier liegen motorische Zellen, deren Achsenzylinder kranialwärts ziehen.

Physiologie.

Unsere Kenntnisse über die Physiologie des Sympathicus gehen in erster Linie auf die klassischen Versuche von $\mathrm{Cla} \mathrm{ude}$ Bernhard zurück, wurden aber wesentlich erweitert, seit man die Sympathicusresektion in die operative Therapie des Morbus Basedowi eingeführt hat. Wir folgen hier den Angaben Jonnesco`s, eines der eifrigsten Vorkämpfer dieser Therapie, doch 
sollen nach Oppenheim nicht alle seine Angaben einwandsfrei sein,

Nach J o n nes co enthält der Halssympathicus:

I. Pupillenerweiternde Fasern für den Musculus dilatator pupillae.

2. Motorische Fasern für die glatten Muskeln der Orbita und des Oberlides, i, e. den glatten, unwillkürlichen Anteil des Musculus levator palpebrae superioris (Musc. tarsalis superior) und den ebenfalls glatten Musculus orbitalis. Dieser (als Rest einer stark entwickelten Muskelschicht, die bei denjenigen Säugetieren sich findet, deren Augenhöhle breit mit der Schläfengrube kommuniziert) überbrückt die untere Orbitalspalte und hindert so den Orbitalinhalt am Zurücksinken.

3. Vasokonstriktorische Fasern für Kopf, Gesicht, Gehirn.

4. Vasodilatatorische Fasern für die Schleimhäute der Wangen, Lipperi, Zahnfleisch, Zunge, Gehirn.

5. Sekretorische Fasern für die Speichel-, Tränen- und Schweißdrüsen des Kopfes.

6. Akzeleratorische Fasern für das Herz.

7. Hemmende Fasern für das Herz.

8. Respiratorische Fasern.

Jementsprechend sind die Zeichen einer Sympathicuslähmung beim Menschen:

I. Verengerung der gleichseitigen Pupille. (Miosis.) Es handelt sich dabei aber nicht um eine vollständige Lähmung des Musculus dilatator iridis, sondern nur um eine Parese; die noch bestehende reflektorische Reaktion der Pupille beweist, daß der Reflexbogen nicht unterbrochen ist und dal, bei der auf Lichteinfall eintretenden Reaktion noch andere, nicht schon im Halsstrang des Sympathicus enthaltene Fasern mitwirken.

2. Verengerung der gleichseitigen Lidspalte. Diese beruht nicht bloß auf einem Tiefstand des obern, sondern auch auf einem Hochstand des untern Lides; eine eigentliche Ptosis, i. e. eine Lähmung des Musculus levator palpebrae besteht nicht, wohl aber des sympathisch innervierten glatten Lidmuskels. Heiligenthal erklärt die Lidspaltenverenge- 
rung so, daß durch die Sympathicuslähmung eine dem natürlichen 'Tonus der Verengerer der Lidspalte entgegenwirkende Kraft in Wegfall gekommen wäre.

3. Zurücksinken des Bulbus. Weniger konstant, auch entwickelt sich diese Erscheinung oft erst allmählich. Sie wird auf Atrophie des Orbitalfettes und auf Lähmung des sog. Musculus orbitalis ( $M$ üller) zurückgeführt. Auch ein Weicherwerden des Bulbus wird zuweilen konstatiert.

4. Erweiterung der Gefäße der entsprechenden Gesichts- und Kopfhaut. Diese Erscheinung wird nicht nur oft vermißt, sondern unter gleichen Verhältnissen eine Verengerung beobachtet. Vielleicht besteht in solchen Fällen Kombination von Reizungs- und Lähmungserscheinungen (O p p e n h e i m) oder es macht sich der regulatorische Einfluß der Gefäßganglien geltend.

In cinzelnen Fällen ergibt sich eine Temperaturdifferenz der Haut zugunsten bald der gesunden, bald der kranken Seite. Das Wesentliche ist aber nicht die Temperaturdifferenz an sich, sondern die Art und Weise der Reaktion auf die Gefäßinnervation beeinflussende Reize (z. B. Körperbewegung. Heiligenthal).

5. Anhydrosis als inkonstantes, vorübergehendes Symptom.

6. Trophische Störungen (Abmagerung) der betreffenden Gesichtshälfte.

I Dic beiden erstgenannten Symptome, Ptosis sympathica und Miosis finden sich bei allen Arten von Lähmungen am konstantesten und werden als Horner'sches Syndrom bezeichnet.

Unsicher sind die Symptome in bezug auf Sympathicusreizung, da die zugrunde liegenden Affektionen fast immer gleichzeitig Bedingungen für die Lähmung von Sympathicusfasern schaffen. Es werden angegeben:

I. Erweiterung der Pupille.

2. Erweiterung der Lidspalte.

3. Gefäßverengerung.

4. Temperaturherabsetzung.

5. Protrusio bulbi.

6. Schwitzen der betreffenden Kopfseite. 
Der experimentellen Reizung und Lähmung des Sympathicus entsprechende Störungen werden beobachtet bei Tumoren der Halsgegend, bei Aortenaneurysmen, bei einfachen, benignen Strumen, bei Struma intrathoracica, bei zufälligen Verletzungen der Halsgegend (Hieb, Stich, Schuß) und endlich bei operativen Eingriffen, als: Resektion und Exstirpation des Sympathicus und seiner Ganglien und endlich bei der gewöhnlichen' Kropfoperation.

A. Sympathicuserscheinungen bei gutartiger Struma.

Erscheinungen von seiten des Halssympathicus, Reizung, Lähmung oder eine Kombination von beiden wurden von den verschiedensten Autoren bei Patienten mit einfacher Struma beobachtet und mit dieser in Zusammenhang gebracht, so daB die Auffassung Wölflers, wonach eine Beeinflussung des Sympathicus durch die gutartige Struma noch wenig sicher gestellt sei, zurzeit wohl nicht mehr zu Recht besteht.

Die jetzt allgemein angenommene Anschauung geht dahin, $\mathrm{da} B$ es tatsächlich der Druck der vergrößerten Schilddrüse ist, der die Sympathicusfunktion stört. Bevor wir diese Auffassung als richtig akzeptieren, dürfte es am Platze sein, zu erörtern, ob nicht anderweitige Erklärungsmöglichkeiten vorliegen, ob nicht etwa eine primär veränderte, krankhafte Funktion der Schilddrüse es ist, die die Lähmung verursacht. Eine solche Annahme würde das fast immer einseitige Vorkommen der Lähmung unverständlich machen. Denn es geht nicht an, der meist einseitig stärkeren Entwicklung der Struma und damit der wohl einseitig veränderten Funktion eine hauptsächlich lokale Wirkung zuzuschreiben. Oder ist etwa die Erkrankung des Sympathicus das Primäre? Dagegen sprechen die Tatsache, da $B$ in den meisten Fällen die Struma zuerst da war und ein gewisses Volumen erreichen mußte, ehe die Sympathicuslähmung auftrat und besonders diejenigen Fälle, bei denen unmittelbar im Anschluß an starke Volumzunahme der Struma Sympathicuslähmung entstand.

Die meisten Beobachter setzen die Druckätiologie daher mit Recht stillschweigend voraus. 
Meistens wird angenommen, daß es eine ganz besondere Form der Struma sei, die den Sympathicus vorwiegend in Mitleidenschaft ziehe: die inthrathoracische. So führt Wölfler das Vorkommen von Sympathicuserscheinungen bei dieser Strumaform an und auch Kocher erwähnt zur Erleichterung der Diagnose auf Struma intrathoracica das Vorkommen von Druckerscheinungen auf die Halsvenen, Rekurrensparese und besonders Lähmung des gleichseitigen Sympathicus, $\mathrm{Da} B$ die in die Thoraxapertur hinabsteigende Struma mit den hier liegenden wichtigen Organen in Konflikt kommt, ist nicht verwunderlich. Denn diese werden im Gegensatz zur Halsregion hier mehr komprimiert als verschoben, da eine Dislozierung nach der Seite nicht möglich ist.

Aber die Sympathicuserscheinungen sind nicht nur dieser Strumaform eigen, sondern es sind manchmal nicht umfang. reiche, flache, mehr oder weniger nach hinten und nach der Seite reichende Strumen, die den Sympathicus gefährden. Gerade diese Fälle sind aber niemals mit Rekurrenslähmung vergesellschaftet, denn der Rekurrens steigt in der Furche zwischen Trachea und Oesophagus verhältnismäßig geschützt liegend aus der Brust auf und liegt dann, sich verzweigend, dem Ringknorpel an. Iei der mehr oder weniger großen Beweglichkeit der Trachea und des Kehlkopfes wird er mit diesen auch dem Druck der Struma ausweichen können, selbst wenn diese hauptsächlich nach hinten und seitwärts wächst. Auch das Gefäßnervenbündel des Halses kann in toto ausweichen, während der Sympathicus, wie wir gesehen haben, auf der Fascia praevertebralis fixiert und dem I)ruck der Struma schutzlos preisgegeben ist. Es mögen aber auch noch die schon besprochenen Varietäten in seinem Verlauf eine Rolle spielen.

Auch die Beschaffenheit des peristrumitischen Bindegewebes und damit auch wohl der Sympathicusscheide ist für das $\mathrm{Zu}$ standekommen der Lähmung von Bedeutung, da derbes und zähes Gewebe das Ausweichen der Organe nur unter einer gewissen Spannung vor sich gehen läßt.

Uber die Seltenheit der Schädigung des Sympathicus bei der einfachen Struma sind die Autoren einig, doch fehlen in der Literatur zahlenmäßige Angaben. 
In unserem Material finden sich unter I 196 von I 895-I9 I 5 operativ behandelten Patienten mit gutartiger Struma I 2 Fälle, d. i. ziemlich genau I Proz., die alle mehrfache Sympathicuserscheinungen zeigten. So außerordentlich selten ist die Sympathicuslähmung also nicht. Ausdrücklich muß hervorgehoben werden, daß es sich in allen unsern Fällen um unzweideutig ausgesprochene Symptome handelte und $\mathrm{da} B$ keine Fälle darunter sind mit nur andeutungsweisem Pupillenunterschied oder nur minimaler Ptose.

Die Sympathicussymptome können entweder allmählich (ent. sprechend einer langsamen Volumzunahme der Struma oder plötzlich in Erscheinung treten: bei akuter Strumaschwellung, wie sie durch intrastrumöse sog. Spontanblutung oder durch akute Blutfüllung des strumösen Venengeflechtes (Blähkropf) bei kongestiven Zuständen vorkommen kann, wie aus nachfolgenden Krankengeschichten $\mathrm{zu}$ ersehen ist.

Allmählich traten die Ėrscheinungen ein in folgenden Fällen:

Fall 1. Frl. A. d. H., 43 Jahre alt. Eingetreten 7. IX. 1904.

Vor 2 Jahren Erstickungsanfall. Atemnot beim Treppensteigen.

$S$ t a t us: Große Patientin von gutem Ernährungszustand. Innere Organe normal. Stimme klar, kein Stridor. Der Hals zeigt rechts vergrößerten Thyreoidallappen von fester Konsistenz. Druck auf letzteren erzeugt Dyspnoe.

Pupillenverengerung rechts, Lidspaltenverengerung rechts.

Operation am 8. IX. I904. Kragenschnitt. Ohne Muskeldurchtrennung Freilegen des rechten, kleinapfelgroßen Lappens. Freimachen des oberen Poles. Unterbindung der Art. thyr. sup. und inf. dextr. Resektion des rechten Lappens. Excision aus dem unteren Teil des linken Lappens.

Heilung per primam. Austritt am 20. IX. I904.

Über Ptosis und Miosis findet sich in der Krankengeschichte keine weitere Notiz. Eine Nachuntersuchung am 20. IX. I9I4 ergab:

Iidspalte rechts ein wenig enger, Pupille rechts kleiner wie links. Patientin gibt an, daß sie nach der Operation oft an halbseitigem Kopfschmerz rechts gelitten habe. Das rechte Auge ermüde schneller als das linke und es stelle sich dann schmerzliches Brennen ein.

F all 2. Frau B. E., 28 Jahre alt. Eingetreten I. III. 1906.

Kropf seit 4 Jahren. Atem- und Schluckbeschwerden. Im letzten Jahr und besonders in den letzten Wochen beträchtliche Zunahme des Kropfes. In retzter Zeit sei die linke Lidspalte zusehends kleiner geworden als die rechte ohne funktionelle Störung des Auges. 
S t a t us: Gut genährte, gesunde Frau. Hals äußerlich wenig verändert, rechts von der 'Irachea eine nicht scharf abgrenzbare, flache, weiche, bewegliche Geschwulst, links ein runder, mehr in der 'Tiefe liegender, pflaumengroßer Knoten, der unter dem Musc. sternocleidomastoideus und unter der Clavicula verschwindet. Er reicht nur bis Mitte Halshöhe hinauf. Psosis links, Miosis links.

Operation am 2. III. Kragenschnitt. I)er rechte Lappen ist oberflächlich, weich, scheint keinen Druck auf die Trachea auszuüben; vorerst wird der linke Lappen freigelegt und luxiert. Fr komprimiert die Trachea stark von links her. Unterbindung der Aa. thyr. sup. und inf. sin. Resektion des linken Lappens. Excision rechts. Präparat: Kolloidstruma.

IIeilung per primam. Austritt am 17. III. I906.

20. VII. I9I4. Man erkennt auf den ersten Blick die gering. gradige, knsmetisch nicht störende Ptosis links und die ebenfalls deutliche Miosis derselben Seite. Patient ist absolut beschwerdefrei.

Fall 3. Frau M. I., 38 Jahre alt. Eingetreten am I7. I. I907.

Struma seit mehr als 13 Jahren. Seit $I^{1 / 2}$ Jahren Bangigkeit, Druckgefühl im Hals, ,aufgelaufenes Gesicht", Dyspnoe, besonders beim Liegen. Vor ca. 3 Wochen heftige Schmerzen auf der rechten IIalsseite, die zuweilen auch ins Ohr ausstrahlten.

Status: MäBig kräftige Patientin mit deutlich cyanotischem Gesicht. Atmung etwas stridorös. I.inke Pupille viel enger als die rechte, deutliche Verengerung der linken Lidspalte. Herzdämpfung nach beiden Seiten etwas verbreitert, Töne rein, hie und da aussetzend.

Am IIalse unterhalb des nicht wesentlich verschobenen Kehlkopfes ein etwa zwetschgengroßer, nicht harter Knoten, der sich nach unten im Jugulum nicht ganz umgreifen läßt und beweglich ist. AuBerdem scheinen die seitlichen, untern Partien des Halses verdickt, wie wenn in der Tiefe noch etwas sitzen würde. Rechts in der Tiefe zwischen den beiden Ansätzen des M. sternocleidomastoideus ist ein harter Knoten fühlbar. Laryngoskopisch normale Verhältnisse.

Operation am 29. I. I907. Kragenschnitt. Derbes Bindegewebe macht das Vorgehen in die Tiefe mühsam. Luxieren des rechten Lappens, der weit nach hinten hinter die Trachea reicht. Ligatur der Art. thyr. sup. dextra. Der linke Lappen reicht sehr weit in die 'Thoraxapertur hinunter, so daB man sein unteres Ende mit dem Finger nicht erreicht. Luxierung und Freilegen sehr schwierig: ligatur der Art. thyr. inf. sin. Die luxierte Struma hat II ufeisenform, der sehr breite Isthmus lag völlig intrathorakal. Totale Resektion des rechten, teilweise des linken Lappens. Blutung ziemlich stark. Patientin war von der Operation ziemlich stark mitgenommen.

2. II. Iupillenverengerung und Ptosis links wie vor der Operation. Nach Ablauf einer interkurrenten Bronchopneumonie am 20. IV. geheilt entlassen. 
16. IV. 1914. Hat an Gewicht zugenommen, im Gesicht keine Cyanose. Ptosis verschwunden, linke Pupille noch eine Spur enger als die rechte.

Fall 4. Frau J. S., 36 Jahre alt. Eingetreten am 25. VII. I9II.

Kropf seit ca. 8 Jahren, im letzten Halbjahr stärkeres Wachstum. Keine wesentlichen Beschwerden.

Status: Gesund aussehende Frau, Herz und Lungen normal. Ptosis links, Miosis links. Deutliche Venenzeichnungen über der Struma und den vordern, obern Partien des Thorax. Gleichmäßige, diffuse Vergrößerung beider Schilddrüsenlappen. Halsumfang: $38,5 \mathrm{~cm}$.

Operation am 27. VII. I9II. Kragenschnitt. Luxieren des rechten Lappens. Ligatur der Art. thyr. sup. und inf. dextr. Resektion des rechten Lappens. Nach Luxieren des linken Lappens Excision eines fast hühnereigroßen Stückes. Die Trachea war von links her säbelscheidenförmig komprimiert. Heilung per primam. Austritt am I I. VIII. Laryngoskopisch normale Verhältnisse.

3I. V. 1915. Lidspalten beiderseits gleich weit, ebenso die Pupillen. Einseitiges Hitzegefühl hat nie bestanden. Keine einseitigen Kopfschmerzen, kein Enophthalmus. Beschwerdefrei.

Fall 5. Frau P. H., 42 Jahre alt. Eingetreten am 7. V. I9I3.

Kleine Struma von Kindheit an, die aber in den letzten 2 Jahren gewaltig zugenommen hat. Starke Atemnot beim Treppensteigen.

$\mathrm{St}$ a $\mathrm{tus}$ : Gut aussehende Patientin. Die rechte Pupille ist etwas größer als die linke, beim Blick nach unten geht das rechte Augenlid nicht ganz so weit mit, wie das linke. Herz und Lungen in Ordnung. Halsumfang: $46 \mathrm{~cm}$. Kugeliger Tumor in der Halsmitte, gut beweglich. Ein etwa zwetschgengroßer Knoten ist neben dem großen links in der Tiefe, ein etwas kleinerer rechts in der Tiefe zu fühlen. Beide sind gut beweglich. Larynx median, Stimmbänder gut funktionierend.

Operation am 8. V. I9I3. Kragenschnitt. Der mediane Tumor gehört zur rechten Seite. Daneben ein tiefer, mehr als zwetschgengroBer Knoten der unter Trachea und Oesophagus reicht und der Luxierung ziemliche Schwierigkeiten bietet. Ligatur der Aa. thyr. sup. und inf. dextr. Resektion des rechten Lappens. Die Trachea ist von rechts her komprimiert. Isolicren des linken, mandarinengroßen Knotens. Ligatur der Art. thyr. sup. sin. Excision des Tumors unter Belassung eines fingerdicken Restes.

Heilung per primam. Laryngoskopisch: Stimmbandfunktion normal. Austritt am 17. V. 19I3.

23. VI. 1914. Linke Pupille enger als die rechte, beide Lidspalten annähernd gleich weit. Nachträglich gibt Patientin an, daß sie auf der linken Gesichtsseite oft ganz kalt gewesen sei, rechts dagegen gleichzeitig feuerrot und heiß, sie habe auf dieser 
Seite stark geschwitzt, die Grenze ging wie mit einem Lineal gezogen scharf durch die Mitte des Gesichtes. Die gleichen Erscheinungen habe Pat. auch noch post operationem gehabt. Sie habe auch öfter Schmerzen auf der linken Halsseite, die sich bis gegen das linke $\mathrm{Ohr}$ hinziehen.

21. V, 19I5. Pat gibt an, daß die oben genannten Beschwerden ungefähr ein Jahr nach der Operation allmählich verschwunden seien, aber auch jetzt noch, wenn sie beim Bergaufgehen sich stark erhitze, werde die rechte Gesichtshälfte hochrot, heiß und schwitze sehr stark, während gleichzeitig die linke Seite blaß und kühl bleibe. Für gewöhnlich hätten beide Gesichtshälften gleiche Farbe. Linke Pupille enger als die rechte, linke Lidspalte nahezu gleichweit wie die rechte. Immerhin ist auf der linken Seite der zwischen dem Sulcus orbitopalpebralis sup. und dem Lidrande gelegene Teil des Oberlides, die Pars tarsalis ca. I mm breiter als rechts. Gesichtsfarbe beiderseits gleich. Kein Enophthalmus. Keine nachweisbare Spannungsdifferenz der beiden Bulbi.

F all 6. Frl. Sch. A., 35 Jahre alt. Eingetreten am 6. III. I9I4.

Struma seit dem I6. Jahr. Seit 2 Jahren offters heftige Schmerzen im Gesicht und im Nacken. Seit einem Monat zunehmende Atemnot, verbunden mit Spannungsgefühl im Kopf, mitunter heftige, nach Kiefer und Nacken ausstrahlende Schmerzen.

Status: Gut genährte Patientin. Herz und Lungen gesund. Normaler laryngoskopischer Befund.

Am Halse rechts apfelgroßer, gut beweglicher, ziemlich harter Strumaknoten; links weicherer, mehr in die Tiefe steigender Knoten von Kindsfaustgröße. Atmung frei.

Linke Pupille enger als die rechte, Lidspalte links enger als rechts.

Operation am 7. III. I9I4. Kragenschnitt. Freilegung, Luxierung und Resektion des kindsfaustgroßen Knotens links. Keilexcision aus dem rechten Lappen. Trachea nicht komprimiert: Heilung per primam. Stimme normal. Entlassung am 2I. III. I9I4.

20. V. 19I5. Starke Ptosis links; die linke Pupille fast um die Hälfte enger als die rechte. Kein einseitiges Hitzegefühl, keine Kopfschmerzen. Dagegen gibt Patientin an, daß das linke Auge sich öfter leicht entzünde, das rechte aber nicht. Momentan besteht in dieser Hinsicht kein Unterschied zwischen beiden Augen.

Fall 7. Frau B. G., 3I Jahne alt. Eingetreten am 3. VIII. I9I4.

Seit Kindheit dicker Hals. Hauptsächlich nachts Atembeschwerden.

Status: Kräftige Frau, sonst gesund. Stimme klar.

Rechte Pupille kleiner, rechte Lidspalte enger als die linke. Kein einseitiges Schwitzen. Rechts kleinfaustgroßer, gut beweglicher Knoten, linker Lappen nicht vergrößert. 
Operation am 4. VIII. I9I4. Kragenschnitt. Nach Unterbindung der rechtsseitigen Arterien Luxierung und Resektion des rechten Lappens unter Belassung eines kleinfingerdicken Restes Strumagewebes.

I7. VIII. I9I4. Entlassung. Laryngoskopisch normale Verhältnisse. Augensymptome noch genau gleich wie beim. Eintritt. Konnte wegen Abreise nicht nachuntersucht werden.

Fall 8. Frau P. H., 45 Jahre alt. Eingetreten am 17. VIII. IgI4.

Struma seit I I Jahren mit der damaligen Gravidität beginnend. Atembeschwerden auch in der Ruhe. Blutandrang zum Kopf.

Status: Schmächtige Frau. Herz und Lungen gesund. Laryngoskopisch normale Verhältnisse.

Linke Pupille etwas kleiner, linke Lidspalte etwas enger als die rechte. Nie einseitiges Schwitzen. Auf der linken Halsseite am vordern Rand des Musc. sternocleidomastoideus zwei hühnereigroße, miteinander zusammenhängende Knoten; rechts ein ebenso großer, der bis zur Clavicula reicht.

Operation am 18. VIII. I914. Kragenschnitt. Freimachen und Luxieren der linken Seite, Unterbindung beider Arterien. Durchtrennen des Isthmus. Resektion des linken I appens unter Belassung eines kleinen Restes Strumagewebes. Excision eines eigroßen Stückes aus dem rechten Lappen. Heilung per primam. Austritt am 23. VIII. IgI 4 .

19. V. 1915. Beide Pupillen genau gleich, gut reagierend. Beide obern Augenlider stehen gleich hoch. Beide Gesichtshälften von gleicher Rötung. I9I4.

F a 11 9. Schüler E. U., I I Jahre alt. Eingetreten am 29. IX.

Seit Mai d. J. Atembeschwerden, die allmählich zunahmen.

St a tus: Kräftiger Knabe, Herz und Lungen gesund.

Pupille rechts enger, Lidspalte rechts enger als links. Es besteht rechts eine kindsfaustgroße, derbe Struma, welche bei tiefer Inspiration im Jugulum vollständig verschwindet und lauten inspiratorischen Stridor erzeugt. Keine Dämpfung über dem Sternum. Keine Vergrößerung des linken Lappens.

Operation am 1. X. IgI4. Kragenschnitt. Freimachen und Luxieren der rechten Seite. Unterbindung beider Arterien. Resektion des rechten Lappens, es bleibt ein kleinfingergroßer Rest Strumagewebe zurück. Der kleinzwetschgengroße linke Lappen wird stehen gelassen. Trachea war von rechts her stark komprimiert. Heilung per primam. Austritt am 9. X. Igr4.

4. VI. I9I 5. Keine Spur von Ptosis und Miosis. Gesichtsfarbe beiderseits gleich. Kein einseitiges Hitzegefühl. Keine Kopfschmerzen. 
Fall Io. Pf. F., 23 Jahre alt. Eingetreten am 8. VII. I9I 5.

Struma seit dem Io. Altersjahr. Atemnot bei strenger Arbeit. Kräftiger, gesunder Mann mit etwas belegter Stimme. Laryngoskopischer Befund normal. Starke Miosis links, starke Ptosis links, zugleich Enophthalmus links. Photographien aus dem 3. und 7. Jahre zeigen, daß diese Sympathicussymptome damals noch fehlten. Sie setzten erst mit dem zunehmenden Wachstum der Struma allmählich ein; eine Aufnahme aus dem 17. Altersjahr läßt Ptosis und Miosis deutlich erkennen.

Auf der linken Halsseite kleinfaustgroßer, verschieblicher, nicht harter, rechts ein etwas weicherer, ca. nußgroßer, leicht verschieblicher Strumaknoten. Trachea nach rechts verschoben. Halsumfang $43 \mathrm{~cm}$.

O peration am 9. VII. I9 I5. Lokalanästhesie. Kragenschnitt. Ohne Muskeldurchtrennung Freimachen und Luxieren der linken Strumaseite, Ligatur der Aa. thyr. sup. et. inf. Durchtrennen des Isthmus. Ablösen des linken Lappens von der Trachea, ein fingerdickes Stück Gewebe bleibt stehen. Excision eines keilförmigen Stückes aus dem rechten Lappen.

Da Patient anamnestisch angab, daß er einseitig schwitze, aber nicht wußte, auf welcher Seite, so wurde er intra operationem speziell beobachtet, wobei sich zeigte, daß er im Gesicht nur rechts schwitzte, während auf der linken Gesichtsseite die Haut absolut trocken war.

13. VII. I915. Enophthalmus zurückgegangen, Ptosis geringer, Miosis noch unverändert.

19. VII. 1915. Ptosis weiter zurückgegangen, Miosis noch wenig verändert. Entlassung.

Ahnliche Beobachtungen werden angeführt von Heiligenthal, Nicati, Seeligmüiler, Holz, Eulenburg, Birch-Hirschfeld, Widal-Abrami, C. Brunner, Dufour, Kocher u. a., aber diese Fälle wurden nicht operiert, oder wenn es geschah, erfahren wir nichts über den Operationsbefund und über die Frage, wie sich die Sympathicussymptome post operationem verhielten. Sie unterscheiden sich also in dieser Hinsicht wesentlich von den unsrigen.

Handelt es sich in unseren vorhergehenden Fällen um langsame und allmählich einsetzende Druckwirkung, also um allmähliches Auftreten der Symptome, so bieten die beiden folgenden Fälle ein ganz anderes Bild: akute Volumzunahme der Struma, akut einsetzende Sympathicussymptome:

a) Durch die recht seltene sog. intrastrumöse Spontanblutung. 
b) Durch pralle Blutanfüllung des reichen klappenlosen Venennetzes infolge Kongestion bei starkem Husten, Bronchitis. Die Volumzunahme kann dabei recht beträchtlich sein. Frank sah z. B. eine Struma, deren kavernöses Gewebe sich so stark mit Blut füllte, daß eine Umfangszunahme des Halses um $5 \mathrm{~cm}$ eintrat.

Fal1 II ${ }^{1}$ ). Frl. Sch. N., 2 I Jahre alt. Eingetreten am I6. VI. rgII.

Dicker Hals seit dem 16. Jahre, die Anschwellung habe aber nur die rechte Seite des Halses betroffen. Seit 3 Jahren hier und da Atemnot. Am I5. VI. I9I I bemerkte Patientin abends bei einem Spaziergang, daß der Hals fest und hart wurde und rechts ein Knollen sich bildete. Die Schwellung wuchs rasch und erreichte in ca. $2 \mathrm{Mi}$ nuten ihren Höhepunkt. Sie bekam Atemnot und Schmerzen, die nach dem Kiefer und Ohr ausstrahlten. Sie wurde heiser und bekam beim Schlucken Schmerzen auf der rechten Seite. Starkes Hitzegefühl im Kopf, und zwar nur rechts. Ein Unterschied der Augen habe vorher nie bestanden.

S t a tus: Mittelgroßes, graziles, etwas anämisches Mädchen. Bewegungen des Kopfes nach rechts gehemmt und schmerzhaft, nach links frei. Die rechte Pupille ist enger als die linke, die rechte Lidspalte enger. Licht- und Konvergenzreaktion der Pupillen beiderseits erhalten. Auf die Frage, ob sie schwitze, macht Patientin die bemerkenswerte Angabe, daß sie nur auf der rechten Gesichtshälfte schwitze, was von der Krankenschwester bestätigt und dahin präzisiert wurde, daß eine scharflinige Grenze bestanden habe, die genau senkrecht durch die Mitte des Gesichtes ging. Dieser Zustand habe früher nie bestanden. Halsumfang $38 \mathrm{~cm}$. Auf der rechten Seite ein gänseeigroßer, wenig druckempfindlicher Tumor von praller Konsistenz.

Operation am 22. VI. rgII. Resektion des rechten Lappens und Excision eines keilförmigen Stückes aus dem linken Lappen in typischer Weise. Präparat: Gänseeigroße Geschwulst, die das Bild einer Struma parenchymatosa zeigt mit einem beträchtlichen zentralen, aus einige Tage altem Blut bestehenden Coagulum. Normaler Wundverlauf.

24. VI. I9II. Miosis dextr. noch vorhanden. ebenso geringgradige Ptosis. Kein einseitiges Schwitzen mehr. Austritt am 3. VII. I9II.

27. VI. I9I4. Ptosis rechts bei genauer Inspektion noch spurweise erkennbar, ebenso ist die Miosis noch spurweise angedeutet.

s) Dieser Fal ist noch in der Arbeit von v, Burg (Beiträge z. klin. Chir.) aufgeführt. 
Auf einer I Jahr nach der Operation aufgenommenen Photographie ist die Ptose noch sehr deutlich erkennbar.

29. V. 19I5. Auch jetzt noch ist bei genauer Beobachtung eine minimale Verengerung der rechten Lidspalte erkennbar. Auch dic rechte Pupille ist eine Spur enger als die linke. Andere Erscheinungen, wie einseitiges Schwitzen, einseitiges Rotwerden kamen nicht mehr vor. Es besteht weder einseitiger Enophthalmus, noch ein merklicher Spannungsunterschied zwischen den beiden Bulbi.

Fall I2, den ich einer Aufzeichnung von Herrn Dr. B runner verdanke. Frl. R. M., 62 Jahre alt. Eingetreten am 5. V. 1910.

Patientin wurde von ihrem Hausarzt behandelt wegen Katarrh und starker Atemnot. $\mathrm{Da}$ die letztere auf die bestehende Struma zurïckgeführt wurde, so wurde Dr. Brunner konsultiert, der am 26. IX. 1910. eine die Atmung stark beengende Struma fand. Auf einer Seite bestand deutliche Ptosis und Miosis. Er riet, erst das Aufhören des Katarrhes abzuwarten und erst dann zu operieren.

Als am 5. V. I9Io Patientin eintrat, war der Katarrh gebessert, die Dyspnoe geringer und die Ptosis und Miosis verschwunden.

Status: Diffuse Vergrößerung beider, besonders aber des rechten Thyreoidallappens. Halsumfang $39,5 \mathrm{~cm}$.

Operation: Typische rechtsseitige Exstirpation, links Resektion. Der linke Lappen griff sehr tief nach hinten und umfaßte die Trachea fast vollständig. Normaler Wundverlauf. Austritt am 3I. III. I9IO.

Von den hier angeführten 12 Fällen sind mit zwei Ausnahmen alle Patienten weiblichen Geschlechts, was aber bei der Tatsache, daß dieses beinahe doppelt so häufig von Kropf befallen ist, wie das männliche, nicht befremdet.

In allen 'unseren Fällen handelt es sich um Lähmungs. symptome, wie denn auch in den in der Literatur angegebenen Fällen diese vorherrschen. Nur in den voneinander recht verschiedenen, auf den ersten Blick paradox erscheinenden Fällen Io und I I findet sich halbseitiges Schwitzen, also Sympathicusreizung. Fall I I zeigte einseitiges Schwitzen auf derselben Seite, auf der Ptosis und Miosis bestanden, also Reizung und Lähmung zugleich. Solche Kombinationen kommen zweifellos vor, gewöhnlich folgen die Lähmungserscheinungen den Reizsymptomen oder sie bestehen nebeneinander. (Oppenheim.) Im Gegensatz hierzu, und auch hier sind Beobachtungsfehler ausgeschlossen, schwitzte Fall io halbseitig auf der den Augensymptomen entgegengesetzten Seite. Hier bestand also auf der Seite des größern Strumaknotens Sympathicuslähmung, auf der entgegen- 
gesetzten Seite Reizung. Wir müssen wohl annehmen, daß in diesem Falle die rechtsseitige, kleinere Struma gerade gro $B$ genug war, um den Grenzstrang zu reizen, zu klein, das peristrumitische Gewebe $z u$ wenig straff, um ihn durch genügend starken Druck zu lähmen. Hatte doch der große linke Knoten durch Verschiebung der Trachea nach rechts dort schon eine Raumverminderung geschaffen, die dem rechten Lappen eine Volumzunahme nur unter Schädigung der Nachbarorgane, des Grenzstranges, ermöglichte. Diese äußerte sich zuerst durch Reizsymptome, aber mit zunehmendem Wachstum des rechten Lappens hätten sich wohl auch hier in nicht allzulanger Zeit Lähmungssymptome eingestellt, wenn nicht die operative Behandlung diesem Verlauf zuvorgekommen wäre.

Über Schmerzen im Innervationsgebjet des Sympathicus ist wenig bekannt. Immerhin ist zu beachten, daB in vier Fällen anamnestisch über halbseitige, oft nach Kiefer und Ohr ausstrahlende Schmerzen berichtet wird. Hierher gehören auch die jedem Operateur bekannten, nach Zähnen, Kiefer und Ohr ausstrahlenden Schmerzen, die bei der Unterbindung der Arteria thyreoidea superior auftreten. Auch die Unterbindung der Art. thyr. inf. wird von manchen Patienten als schmerzhaft empfunden.

Alle unsere Patienten sind strumektomiert worden und jedesmal gab die Operation der äußeren Untersuchung, die auf der Scite der Sympathicusläsion den größern oder mehr nach hinten oder unten reichenden Knoten gefunden hatte, recht; wohl der beste Beweis für die Irucktheorie. Demgemäß wurde auf der Seite der Sympathicuslähmung jeweils die Resektion ausgeführt, während man sich auf der andern Seite mit der Excision eines keilförmigen Stückes begnügen konnte. Nur bei Fall 4 wurde die Resektion auf der der Lähmung entgegengesetzten Seite (rechts) gemacht, aber auch dort zeigte es sich, daß derjenige Strumalappen, der in erster Linie der Entfernung bedurfte, auf der linken Seite saß, denn außer dem Grenzstrang war auch die Trachea linkerseits komprimiert. Anschließend an diesen Fall dürfte der Rat am Platze sein: wenn bei einer mit Sympathicussymptomen vergesellschafteten Struma die äußere Untersuchung keine Sicherheit gibt, auf welcher Seite der größere 
Tumor sitzt, so möge man die der Sympathicusläsion entsprechende Seite zuerst in Angriff nehmen.

Wie verhalten sich nun die Sympathicuserscheinungen, wenn der sie verursachende Druck behoben ist, also nach der Kropfoperation? Das zeigen am besten die Augensymptome. Diese bilden sich manchmal rasch zurück, sobald die Schädlichke:t behoben ist, können aber in andern Fällen trotzdem noch jahrelang bestehen bleiben.

So waren bei Fall I 2 nach zehn Tagen die Lähmungserscheinungen verschwunden, noch ehe operiert wurde (hier genügte die Rückbildung des Kongestivzustandes), bei Fall 9 waren sechs, bei Fall 8 neun Monate, bei Fall 4 vier Jahre, vielleicht auch weniger zur Rückbildung der Lähmungserscheinungen nötig, während andererseits Fall I noch zehn Jahre post operationem geringgradige Ptosis und deutliche Miosis zeigte.

Immerhin pflegt im Laufe der Zeit die Ptosis zuerst zu verschwinden, die Miosis bleibt viel länger bestehen und bildet s.ch noch langsamer zurück. So war bei Fall 5 nach dre:zehn Monaten die Ptosis noch angedeutet, aber auch nach zwei Jahren noch nicht völlig verschwunden, die Miosis war nach dieser Frist noch deutlich. Fall I I (Intrastrumöse Spontanblutung) zeigte nach drei Jahren eine Spur von Ptosis, die Miosis zwar geringer, doch noch deutlich, trotzdem die Sympathicuskompression nur sieben Tage gedauert hatte. Ein Jahr später war ein Rest von Ptosis nur bei scharfer Beobachtung zu erkennen, während die Miosis unbedeutend, doch gut erkennbar war. Bei Fall 3 war nach sieben Jahren die Ptosis verschwunden, die Miosis noch angedeutet. Fall 6 zeigt nach dreizehn Monaten die Augensymptome noch unverändert. Auch Fall Io ist in dieser Hinsicht instruktiv. Man konnte hier trotz der kurzen Beobachtungsfrist erkennen, daß die Ptosis post operationem rasch sich gebessert hatte, während die Miosis nur unwesentlich beeinflußt worden war.

B. Sympathicuslähmungen nach Strumektomie.

Noch seltener als im Gefolge einer einfachen, gutartigen Struma treten Sympathicuserscheinungen im Anschluß an die Operation von gewöhnlichen Strumen auf. Solche Fälle erwähnen 
Kappeler, v. Bruns, Reverdin, Kolaczek, Eisels. berg u. a. Auch hier handelt es sich in der Regel um eine Lähmung. Nur in Kappelers Fällen, in den ersten, wo überhaupt nach einer Kropfoperation Sympathicuserscheinungen beobachtet wurden, scheint es sich nicht um eine Lähmung, sondern um eine Reizung zu handeln, weshalb wir diese beiden Fälle hier anführen.

1. Susanna B., I8 Jahre alt, kam am 23. I. 1866 ins Spital. Hatte schon als Kind einen dicken Hals und suchte $z u$ wiederholten Malen wegen dieses Leidens ärztliche Hilfe. Seit 2 Jahren stärkere Beschwerden namentlich beim Schlucken, so zwar, daß sie feste Bissen kaum hinunter brachte. Fortwährend mäßige, bei raschem Gehen und anstrengender Arbeit starke Dyspnoe. Vom rechten Lappen der Thyreoidea ging ein ungefähr eigroßer Geschwulstknoten aus, der, ziemlich frei beweglich, weder auf Trachea noch Ösophagus drückte. Der linke Lappen der Schilddrüse aber war in einen über mannsfaustgroßen Tumor verwandelt, der tief unter das Sternum reichte und bei dessen Kompression die Kranke sofort starke Dyspnoe bekam und dann nur noch mit Mühe Wasser hinunterschlucken konnte.

Exstirpation dieser Geschwulst am 19. I. mit doppelter Unterbindung der zuführenden Gefäße. Es mußten 22 Ligaturen angelegt werden und die Kranke verlor im ganzen ca. eine Unze (35 Gramm) Blut. Die Durchmesser der Geschwust waren I2, 9, $5 \mathrm{~cm}$. Die Heilung der Wunde machte keine großen Schwierigkeiten, schon am I7. II. war die Kranke fieberfrei. Mehrmals hatte sie heftige linksseitige Kopfschmerzanfälle und die Erweiterung der linken $\mathrm{Pu}$ pille, die schon am 2o. I., am Tage nach der Operation, bemerkt wurde, blieb noch nach dem am 3. III. erfolgten Schluß der Wunde.

2. Magdalena Sp., 25 Jahre alt, ein geistig ziemlich beschränktes, anämisches Frauenzimmer, soll schon im 14. Lebensjahr im Besitz einer ziemlich großen Kropfgeschwulst gewesen sein. Damals und im Jahre 186I machte sie wochenlang Einreibungen mit Jodsalbe, doch mit wenig Erfolg. In den letzten Jahren langsames, aber stetiges Wachsen des Kropfes und bedeutende Dyspnoe, die ihr jede schwere Arbeit verbot. Beim Eintritt ins Spital fand ich eine apfelgroße, rundliche, sich glatt anfühlende, fest und median aufsitzende Geschwulst. Druck auf dieselbe macht Dyspnoe.

Ausschälung derselben am 13. I. I868. Nach Freilegung der Geschwulst stellte sich heraus, daß dieselbe vom rechten Lappen der Drüse ausging und die Trachea schief von rechts vorne nach links hinten komprimierte, und da der Processus pyramidalis auch in zwei walnußgroße Tumoren verwandelt war, die der Luftröhre links aufsaßen, war dieselbe fest zwischen zwei Geschwulstmassen 
eingekeilt. Es wurde der ganze rechte Lappen und der vergrößerte Processus pyramidalis entfernt. Die starke Blutung aus einer kleinen Arterie konnte nur mit vieler Mühe durch Umstechung gestillt werden. Vom 21. III. (?) an war die Patientin bleibend fieberfrei und die Heilung der großen Wunde ging von dieser Zeit an sehr rasch vor sich und war am 20. III. vollendet, so daß Patientin am 28. entlassen werden konnte.

Am 2. Tage nach der Operation erfolgte eine kleine Nachblutung, und auch hier beobachtete ich das schon in der letzten Krankengeschichte erwähnte Phänomen der Pupillendifferenz. Es war nämlich die rechte Pupille nicht bleibend, wie im letzten Fall, sondern nur etwa I4 Tage lang nach der Operation, aber auch in diesen I4 Tagen nicht immer konstant, beträchtlich weiter als die linke.

$\mathrm{Zu}$ diesen Fällen bemerkt Kappeler: ,Nirgends fand ich die Beobachtung verzeichnet, die ich zweimal machte, daß nämlich die Pupille auf der operierten Seite bleibend oder vorübergehend sich erweiterte. Die nächstliegende Erklärung ist wohl die, daß der in der Operationswunde liegende Grenzstrang des Sympathicus in Mitleidenschaft gezogen wird und durch entzündliche Reizung desselben die Pupillenerweiterung zustande kommt".

Was in diesen beiden Fällen vorgelegen hat, ist schwer zu sagen. Sie stehen mit unsern Erfahrungen und denjenigen aller andern Operateure in Widerspruch. Die Angaben sind aber so positiv und von einem so guten objektiven Beobachter gemacht, daß wir sie als richtig ansehen und die Erklärung Kappelers akzeptieren müssen. Wir müssen bedenken, daß die beiden Fälle noch in der vorantiseptischen Zeit liegen, wo die Strumen nicht systematisch nach genauen anatomischen Grundsätzen, sondern atypisch wie irgendeine Geschwulst exstirpiert wurden und wo immer erst nach langwieriger Eiterung Heilung eintrat.

Unser eigenes Material weist unter I 96 operierten Strumafällen sechs mit konsekutiver Sympathicusläsion auf, also $0,5 \circ$ Proz. Mit einer Ausnahme zeigten alle Fälle normalen Heilungsverlauf und ausschließlich Lähmungssymptome.

Fall I3. Frl. Sch. B., 33 Jahre alt. Eingetreten am 27. III. I9I3. Schon als Kind Struma, die allmählich, in letzter Zeit stärker wuchs. Sie bekam Druckgefühl im Hals, Atembeschwerden, Herzklopfen. 
Status: Mittelgroße, kräftige Patientin. Pupillen beiderseits gleich, gut reagierend. Auf der rechten Halsseite ca. apfelgroßer, gut beweglicher, zwischen Trachea und Sternocleidomastoideus gelegener Tumor von weicher Konsistenz. Links ebenfalls kleinerer Knoten fühlbar. Kehlkopf und Trachea median. Übrige Organe gesund.

Operation am 28. III. I9I3. Modif. Kragenschnitt. Eingehen in der Medianlinie, Durchtrennen der fibrösen Kropfkapsel. Das peristrumitische Gewebe ist äußerst straff, das Freilegen und Luxieren der Struma stark erschwerend. Ohne Durchtrennung von Halsmuskeln wird erst der rechte, obere Pol freigelegt und die Art. thyr. sup. ligiert und durchtrennt. Das Aufsuchen der Art. thyr. inf. bereitet wegen des straffen Bindegewebes Schwierigkeiten. Unterbindung derselben in continuitate. Durchtrennen und Ablösen des Isthmus von der Trachea und Resektion unter Zurücklassung eines kleinen Kapselrestes zur Schonung des Rekurrens. Die Luxierung des linken Lappens gelingt leichter. Entfernung des vergrößerten Teiles durch Keilexcision. Versorgung des Restes mit Katgutknopfnähten. Glasdrain. Steriler Verband. Präparat: Kolloidstruma.

30. III. I9I3. Ptosis rechts, Miosis rechts. Eine Woche später bestehen diese immer noch, erstere scheint eher stärker geworden $\mathrm{zu}$ sein.

18. IV. I9I3. Die Augensymptome sind unverändert. Patientin war darob sehr unglücklich. Sonst geheilt entlassen. Sie ging in der Folge verschiedene Augenärzte vergeblich um Hilfe an.

26. VI. I9I4. Ptosis und Miosis verschwunden.

Fall I4. Frl. B. E., 25 Jahre alt. Eingetreten am 28. III. I9I3.

Struma seit sechs Jahren, in letzter Zeit Umfangszunahme. Bei strenger Arbeit Atemnot, Herzklopfen, Bangigkeit. Weder Schluckbeschwerden, noch Erstickungsanfälle.

Status: Gut genährte, kleine Patientin. Pupillen beiderseits gleich, kein einseitiges Schwitzen. Halsumfang $37 \mathrm{~cm}$. Auf der rechten Halsseite orangegroßer, derber, dem rechten Lappen entsprechender Knoten. Links ein weicherer, kleinapfelgroßer, beweg. licher Tumor. Laryngoskopisch normale Verhältnisse. Keine laryngotracheale Verschiebung.

Operation am 29. III. I9I3. Kragenschnitt. Medianes Eingehen auf die Struma. Luxierung des rechten derben Lappens ist schwierig, da die peristrumitischen Bindegewebslamellen äußerst zähe sind. Abtrennen des obern Poles. Aufsuchen und Unterbinden der Art. thyr. inf. aus dem gleichen Grunde nur schwer ausführbar. Durchtrennen des Isthmus und Ablösen von der Trachea. Resektion des rechten Lappens unter Schonung des deutlich sichtbaren N. recurrens. Luxierung des linken, kleinern Lappens, der die Trachea säbelscheidenförmig komprimiert, bedeutend leichter. Keilförmige Ex- 
Über Störungen von seiten des Halssympathicus bei einfacher Struma. usw. 4 I 5

cision. Blutstillung. Glasdrain. Hautklammern. Steriler Verband. Nach der Operation Ptosis und Miosis rechts.

3I. III. I9I3. Fieber, Eiterung. Zehn Tage später Pupillendifferenz noch vorhanden, geringe Ptosis rechts. Eitersekretion nur noch unbedeutend.

I8. IV. I9I3. Augensymptome verschwunden. Angina lacunaris. Flecken auf der Streckseite der Unterschenkel nach Art des Erythema nodosum.

9. V. I9 3. Geheilt entlassen. Stimme normal.

Fal1 15. Frl. L. E., 40 Jahre alt. Eingetreten am 4. VII. I913.

Struma seit zehn Jahren. Stärkeres Wachstum seit einem Jahr. Keine Beschwerden.

Status: Kräftige Patientin mit gesunden Organen. Pupillen beiderscits gleich. Keine Ptosis, kein einseitiges Schwitzen. Halsumfang $39 \mathrm{~cm}$. Rechts von der Medianlinie eine mandarinengroße, kugelige Cyste; der ubrige rechte Lappen sonst als wenig vergrößert zu fühlen. Links ca. kleinfaustgroßer Tumor. Larynx und Trachea nicht verschoben.

Operation am 5. VII. I9I3. Modif. Kragenschnitt. Medianes Eingehen ohne Muskeldurchtrennung. Freimachen und Luxieren der rechten Seite. Unterbindung der Art. thyr. sup., dann der dicken inferior in continuitate. Durchtrennen des Isthmus. Ablösung des rechten Lappens von der Trachea unter Zurücklassung eines fingerdicken Restes Strumagewebes am Hilus. Auf der linken Seite Herausschälen zweier Cysten unter ziemlich starker Blutung. Blutstillung, Glasdrain, stcriler Verband. Präparat: Struma colloides.

Post operationem Ptosis und Miosis rechts. Normale Heilung.

I2. VII. 1913. Entlassung. Noch ziemlich starke Ptosis und Miosis.

22. VI. 1914. Ptosis noch angedeutet, Miosis noch deutlich. Kein Zurücksinken des Bulbus, keine Dystrophie. Patientin war wegen dieser operativen Sympathicuslähmung sehr ungehalten, nicht weil das tiefer stehende Oberlid sie beim Blick nach oben hinderte, sondern hauptsächlich des ästhetischen Defektes wegen. Sie suchte einen Augenarzt auf, der Lähmung eines Nerven diagnostizierte, aber einen kausalen Zusammenhang mit der Strumaoperation ablehnte. Pat. bcobachtete selbst, wie die Ptosis allmählich sich zurückbildete. Einseitiges Erröten, Schwitzen sei nie vorgekommen.

20. V. I915. Das rechte Oberlid eine kaum erkennbare Spur tiefer stchend als das linke, auch ist die rechte Pupille etwas enger als die linke. Gesichtsfarbe beiderseits gleich, keine einseitigen Kopfschmerzen. Beschwerdefrei.

Fall i6. Frau Ch. M., 40 Jahre alt. Eingetreten am 9. VIII. I913. 
Struma seit dem I8. Lebensjahr. März I9I3 mehrere Hämoptoen. Dyspnoe beim Treppensteigen und Herzklopfen.

Status: Recht gutes Aussehen, sonnenverbranntes Gesicht. Affektion des rechten Apex pulm. Halsumfang $35,5 \mathrm{~cm}$. Orangegroßer Tumor in Halsmitte, dem rechten Lappen angehörig und nach rechts in die Tiefe sich fortsetzend. Linker Lappen leicht vergrößert. Trachea und Larynx stark nach links verdrängt. Pupillen und Lidspalten beiderseits gleich.

Operation am I2. VIII. I9I3. Modif. Kragenschnitt. Freimachen und Luxieren der rechten Seite. Nach Unterbindung der Arterien Freimachen des oberen und unteren Poles, Durchtrennen des Isthmus und Ablösen des rechten Lappens von der Trachea. Es bleibt ein kleinfingergroßes Stück Strumagewebe stehen. Keilexcision aus dem linken Lappen. Die Trachea ist von rechts her komprimiert. Blutstillung, Glasdrain, Klammern, steriler Verband. Präparat: Kolloidstruma.

Noch im Operationssaal, als man den Verband anlegte, bemerkte man starke Ptosis und Miosis rechts.

21. VIII. I9I3. Geheilt entlassen. Augensymptome noch angedeutet, Stimme normal, laryngoskopisch normal.

I9. VI. I9I4. Starke Miosis rechts, ebenso deutliche, wenn auch geringe Ptosis rechts. Patientin klagt über starkes, sehr lästiges Hitzegefühl auf der rechten Gesichtsseite. Diese sei oft stärker gerötet als die linke. Häufig rechtsseitiges Kopfweh. Bei der Inspektion der Patientin fällt besonders auf, daß die rechte Wange voller, wie geschwollen, ist als die linke. Die links vorhandene Grube in der Wange ist rechts völlig verstrichen.

29. V. I9I5. Ganz geringgradige Ptosis rechts. Miosis rechts deutlich, wenn auch nicht auffällig. Die rechte Wange ist noch immer voller als die linke. Auch jetzt noch fehlt rechts die in der linken Wange vorhandene seichte Grube. Auch ist die rechte Wange leicht gerötet, während die linke blaß ist. Dieser Unterschied sei, wie Pat. angibt, besonders zur Zeit der Menstruation auffallend, aber auch bei jeder zu Erhitzung führenden Anstrengung. Einseitiges Schwitzen besteht nie. Die Kopfschmerzen, über die Pat. schon früher geklagt, bestehen noch. Sie betreffen hauptsächlich die rechte Schläfengegend und strahlen von da gegen die rechtsseitige Occipitalgegend aus. Pat. beklagt sich auch über Schwäche des rechten Auges, die darin bestehe, daß nach kurzem Lesen oder Nähen dieses überfließe und dann ausgewischt werden müsse. Spannungsunterschied der Bulbi besteht nicht. Im allgemeinen haben die Beschwerden aber eher ab, als zugenommen.

Dieser Fall, bei dem es sich um eine reine Lähmung handelt, ist insofern bemerkenswert, als sich hier die Ernährungsstörung der Gewebe im Sinne einer Hypertrophie geltend macht, 
und zwar ist sie deutlich und kann der Beobachtung nicht entgehen. Sie fiel auch meinem Mitassistenten, Dr. St ocker, der die Patientin sah, sofort in die Augen. Die ungezwungenste Er klärung ist wohl die, daß hier infolge der Lähmung Gefäßerweiterung und damit bessere Durchblutung und Ernährung der Gewebe eingetreten ist. Auch dürfte es sich um Fettansatz. handeln, dd die leichte Schwellung keineswegs Ödemcharakter hat.

Die Gefäße sind wie alle vegetativen Organe (mit Ausnahme der Schweißdrüsen, der Haarwurzeln, und eines Teiles der Gefäßmuskulatur der Eingeweide mit ausschließlich sympathischer Innervation) doppelt innerviert. Dabei besteht vielfach Antagonismus zwischen dem autonomen und sympathischen System in dem Sinne, daß da, wo der Sympathicus die Tätigkeit fördert, sie durch das autonome System gehemmt wird und umgekehrt. In unserm Falle ist wohl durch Wegfall der vasokonstriktorischen Sympathicuswirkung der Antagonismus des autonomen Systems voll zur Geltung gekommen.

Für die Annahme einer Thrombose der Vena facialis, die ähnliche Erscheinungen machen könnte, liegt keine Veranlassung vor, da Schwellungen, die nach Unterbindung der Vene gelegentlich auftreten, bald wieder $\mathrm{zu}$ verschwinden pflegen.

Fall I7. Herr A. S., 27 Jahre alt. Eingetreten am I. IV. I9I4.

Struma seit dem Io. Altersjahr. Atembeschwerden beim schnellen Gehen, Turnen usw.

Status: Kräftiger Patient mit gesunden Organen. Stimme klar. Pupillen beiderseits gleich, keine Ptose, kein einseitiges Schwitzen. Am Halse rechts außen, unterhalb des Kehlkopfes ein vergrößerter Strumaknoten, der über die Trachea etwas nach links und bis zum Manubrium sterni reicht; er ist gut beweglich, sein unteres Ende abtastbar.

Operation am 3. IV. I9I4. Modif. Kragenschnitt. Ohne Muskeldurchtrennung Freimachen und Luxieren der rechten Seite. Äußerst derbes und zähes peristrumitisches Gewebe. Nach Arterienunterbindung Resektion des rechten Lappens. Ein kleinfingerdicker Rest Strumagewebe bleibt an der Eintrittsstelle der Art. thyr. inf. bestehen. Trachea von rechts her stark abgeplattet. Blutstillung, Glasdrain, steriler Verband. Post operationem Ptosis und Miosis rechts. Normaler Wundverlauf.

II. IV. I9I4. Geheilt entlassen, Augensymptome unverändert. Ebenso bei einer Nachuntersuchung I4 Tage später. Stimme klar, dagegen klagt Pat. über Kopfschmerzen. 
20. VI. I9I4. Ptosis und Miosis noch gleich. Beim Blick nach oben stört das paretische herabhängende Oberlid. Sonst keine Beschwerden.

3I. V. I9I5. Rechte Lidspalte noch immer etwas enger als die linke, jedoch nicht mehr so, daß der Blick nach oben behindert würde. Auch ist die rechte Pupille etwas enger wie die linke. Kein einseitiges Hitzegefühl. Keine Beschwerden.

Fall 18. Frau M. S., 40 Jahre alt. Eingetreten am 18. V. 19I4. Struma seit 20 Jahren. Atemnot beim Treppensteigen.

Status: Kräftige, gesunde Patientin mit klarer Stimme. Pupillen beiderseits gleich, keine Ptosis, kein einseitiges Schwitzen. Am Halse links von der Mittellinie eine faustgroße, elastische, gut bewegliche Strumageschwulst, vom linken Lappen ausgehend und mit breitem Isthmus über die nach rechts verschobene Trachea in einen rechten, steinharten, nußgroßen Knoten übergehend.

Operation am 19. IV. I9I4. Modif. Kragenschnitt. Freimachen und Luxieren der linken Seite gelingt sehr leicht. Ligatur der linken Aa. thyr. sup. und inf. Vom Isthmus her Abtrennen des linken Lappens und Resektion mit Zurücklassung eines ca. fingerdicken Stückes Strumagewebes. Rechts Keilexcision, auch hier bleibt ein fingerdickes Stück Strumagewebe stehen. Blutstillung. Hautklammern, steriler Verband. Präparat: Kolloidstruma von $3 \mathrm{I} 8 \mathrm{~g}$ Gewicht. Nach der Operation Ptosis und Miosis links.

28. V. IgI4. Nach normalem Wundverlauf geheilt entlassen. Ptosis besteht noch, ist aber entschieden zurückgegangen. Miosis noch unbedeutend.

I5. VI. I9I4. Die Augensymptome nicht merklich gebessert. Patientin gibt an, daB das Oberlid sie beim Blick nach aufwärts behindere. Ein deutlich erkennbarer Spannungsunterschied der Bulbi besteht nicht.

7. VI. I9I 5. Ptosis stark zurückgegangen und nur bei genauem Zusehen noch deutlich erkennbar, die Miosis noch fast unverändert. Patientin ist absolut beschwerdefrei. Gesichtsfarbe beiderseits gleich. Kein einseitiges Schwitzen, keine Kopfschmerzen.

Unsere 6 Fälle lassen sich in zwei Gruppen gliedern:

I. Die Fälle I3, I4, I7 mit sehr derbem, zähem peristrumitischen Bindegewebe.

II. Die Fälle I 5, I6, I8 mit normalem oder (Fall I8) zartem peristrumitischen Gewebe, das ein Auslösen der Struma besonders leicht gestattet.

Sprechen wir bei Gruppe I der Beschaffenheit des Bindegewebes für das Zustandekommen einer operativen Lähmung 
eine gewiß vorhandene ursächliche Bedeutung $\mathrm{zu}$, so ist für Gruppe II diese Ursache fraglich.

Derbes Bindegewebe erschwert das Luxieren der Struma bedeutend, es kommt leichter als sonst zu Blutungen, die nicht sofort stillbar sind. Das Gewebe wird dadurch blutig suffundiert und die klare Übersicht geht beim Vordringen in die Tiefe verloren. Folge davon ist das Bestreben, sich vom gefährdeten Rekurrens möglichst fern zu halten und die Art. thyr. inf. möglichst weit lateral $\mathrm{zu}$ isolieren und $\mathrm{zu}$ ligieren. Daß dabei der Grenzstrang, besonders wenn er ganz vor der Arterie liegt oder sein vorderer, den Schlitz bildender Ast oder gar das Ganglion cervicale medium in die stumpf isolierende Pinzette gerät, dabei gezerrt oder zerrissen wird, ist verständlich. Wenn in solchen Fällen der Operateur vor die Möglichkeit einer Rekurrens- oder Sympathicusläsion gestellt ist, so wird er wohl immer das Risiko der letzteren auf sich nehmen, wenn er es überhaupt in den Bereich seiner Erwägungen zieht.

Auch bei Fällen mit zartem Bindegewebe, deren Operation überhaupt keine Besonderheiten bietet und glatt vonstatten geht, kann es ausnahmsweise zu Sympathicusschädigung kommen.

Es besteht aber noch eine andere Erklärungsmöglichkeit, die bestärkt wird durch die Tatsache, daß; unsere Beobachtungen, die sich über einen Zeitraum von zwei Jahrzehnten erstrecken, alle in den Jahren 19I3 und I9I4, also zeitlich auffallend eng beisammen liegen. Das ist der Zeitpunkt, da wir zur Anwendung der Lokalanästhesie nach $\mathrm{Braun}$ übergingen, während vorher die einfache Infiltration der Schnittlinie geübt wurde. Das bei Braun beschriebene Verfahren gründet sich auf die Tatsache, daß die Cervikalnerven in der Gegend der Querfortsätze des dritten bis fünften Halswirbels ziemlich nahe beisammen liegen. Man sticht daher an zwei Stellen von der Seite her auf die Querfortsätze ein und infiltriert typisch die ganze, durch die beiden Einstichpunkte, die Querfortsätze und die Haut begrenzte Gewebsschicht. Man kommt dabei weder mit dem Gefäßnervenstrang, noch mit dem Sympathicus in Kollision. Wenn nun eine weniger geübte Hand die Querfortsätze nicht trifft, sondern vor ihnen zu tief einsticht, liegt ein zufälliges Auftreffen auf den Grenzstrang nicht außer Bereich der Möglichkeit. Wird der- 
selbe mit dem Anästheticum nur infiltriert, so müssen die Lähmungserscheinungen nur vorübergehende sein, wenn aber die Nadel in den Nerven eingedrungen und dabei Fasern zerstört hat, kann naturgemäß die Lähmung länger anhalten, oder sogar dauernd sein.

Dauer und Prognose der Lähmungssymptome.

Seit Einfuhrung der Sympathicusresektion in die Therapie des Morbus Basedowi hatte man Gelegenheit, sich von der Ungefährlichkeit der Folgen zu überzeugen. Hier, wie bei der zufälligen Verletzung während der Kropfoperation, treten die Augensymptome, Lidspalten- und Pupillenverengerung regelmäßig in Erscheinung. Sind sie auch nicht lebensgefährlich, so bilden sie, weil einseitig, eine beachtenswerte kosmetische Störung, über die namentlich weibliche Patienten manchmal recht ungehalten sind. Sie gehen dann oft von einem Augenarzt zum andern, finden aber nirgends Hilfe, denn da es sich nicht um eine reine Ptosis handelt, kommt eine Operation nicht in Frage.

Hand in Hand mit der kosmetischen Störung der Ptose geht die Klage, daß das tiefer stehende Oberlid den Blick nach oben behindert, was manchmal recht lästig ist. Zum Tragen einer Ptosisbrille, die die Aufgabe hätte, das Lid zu stützen, oder gar

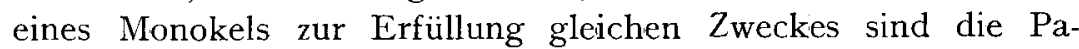
tienten kaum zu bewegen.

Bemerkenswert ist auch die Angabe (Fall I6), daß bei feinern, die Augen anstrengenden Arbeiten wie Nähen, Lesen das der Seite der Sympathicusläsion entsprechende Auge überfließe. Dals weist auf eine Störung der Tränensekretion hin, die zwar bei normalen Ansprüchen richtig funktioniert, aber unter gewissen Bedingungen krankhaft arbeitet. Diese Erscheinung wäre in Parallele zu setzen mit den Fällen, wo die Gefäßinnervation des Gesichtes für gewöhnlich normal ist, aber bei erhöhten Ansprüchen (Bergsteigen usw.) gestört ist. Auch Fall I zeigt eine ähnliche Tränenstörung. Anomalien in der Tränensekretion gehören zwar nicht zum klassischen Bilde der Sympathicuslähmung, sind aber gemäß der sympathischen Innervation der Tränendrüse nicht von der Hand $z$ u weisen.

Recht unangenehm und hartnäckig sind die im Gefolge der 
halbseitigen Lähmung auftretenden migräneartigen Kopfschmerzen, wie sie schon bei der Drucklähmung erwähnt wurden.

Wenn, wie bei Fall r6, halbseitige Rötung, Hitzegefühl und gar eine auffallende trophische Störung im Gesicht sich mit den schon genannten Beschwerden verbinden, dann ist die Lähmung keine gleichgültige Sache mehr, und man muß sich ernstlich fragen, wie solche Fälle zu vermeiden sind.

Die eventuell auf das Konto der Lokalanästhesie fallenden Lähmungen sind durch richtige Ausführung derselben ohne weiteres vermeidbar. Anders die operativen Fälle. Wie dem geübtesten Chirurgen einmal eine Rekurrenslähmung passieren kann, so können auch Verhältnisse auftreten, bei denen man das Vermeiden einer Sympathicusverletzung nicht mehr sicher in der Hand hat. Man denke nur an jene Fälle mit derbem, peristrumitischem Gewebe. Immerhin soll man beim Aufsuchen der Art. thyr. inferior recht sorgfältig vorgehen, die Arterie genau isolieren und möglichst für sich unterbinden, denn der Sympathicus liegt in unmittelbarer Nähe.

Über die Dauer der Lähmungssymptome finden sich in der Literatur keine Angaben, doch lassen unsere Beobachtungen den Schluß zu, daß sie in einzelnen Fällen sich zurückbilden können. Wie bei der Drucklähmung, so pflegt auch hier die Ptosis in erster Linie sich zurückzubilden, während die Miosis noch lange Zeit nachher bestehen kann. Diese Rückbildungsmöglichkeit ist naturgemäß von der Art und Größe der Sympathicusläsion, über die wir uns bei der Operation selten eine Vorstellung machen können, abhängig.

Bei Fall I 4 waren Ptosis und Miosis nach drei Wochen, bei Fall $x_{3}$ nach dreizehn Monaten verschwunden. Fall I 5 zeigt nach fast zwei Jahren die Ptosis nur noch angedeutet, die Miosis eher deutlich, und Fall 16 nach einundzwanzig Monaten geringe Ptosis, deutliche Miosis. Die Fälle 17 und 18 zeigten sich nach zwei, bzw. einem Monat unverändert, ein Jahr später aber bedeutend gebessert.

Nach diesen Erfahrungen scheinen in den meisten Fällen die Sympathicussymptome mit der Zeit sich zurückzubilden. Es ist aber nicht ausgeschlossen, daß sie in einzelnen Fällen zeit- 
lebens bestehen bleiben können, welche Frage aber an Hand des vorliegenden Materials nicht zu entscheiden ist.

$$
\text { Zus a mmenfassung. }
$$

I. Die einfache, benigne Struma kann durch Druck den Grenzstrang des Sympathicus beeinträchtigen.

2. Nach Exstirpation der betreffenden Kropfhälfte bilden sich in einem Teil der Fälle die Sympathicussymptome zurück, in andern Fällen bleiben sie bestehen. Von den Augensymptomen 'schwindet zuerst die Ptosis und erst nachher die Miosis.

3. Der Sympathicus kann bei einfacher Kropfoperation verletzt werden. Derbe, peristrumitische Verwachsungen begünstigen das Zustandekommen der Verletzung.

4. In der Regel handelt es sich dabei um Lähmungssymptome. Auch hier sind sie einer Rückbildung fähig. Zuerst schwindet die Ptosis und dann die Miosis. .

5. Die operative Sympathicuslähmung bildet eine beachtenswerte Störung, die durch sorgfältiges Vorgehen im Gebiet der Arteria thyreoidea inferior nach Möglichkeit zu vermeiden ist.

\section{Literaturverzeichnis.}

I. T. Drobnik, Topogr. anat. Studien über den Halssympathicus mit besonderer Berïcksichtigung des Terrains der Kropfoperationen. Arch. f. Anat. 1887 .

2. A. Wölfler, Die chirurgische Behandlung des Kropfes. Langenb. Arch. XL. Bd. 29 u. 3 o.

3. v. Eiselsberg im Handbuch der praktischen Chirurgie.

4. Derselbe, Die Krankheiten der Schilddrüse. D. Chr. 38.

5. Oppenheim, Lehrbuch der Nervenkrankheiten. I9I3.

6. Heiligenthal, Beiträge zur Kenntnis der Lähmungen des Halssympathicus. Arch. f. Psych. XXXII.

7. P. v. Bruns, Die Entwicklung der modernen Behandlung des Kropfes.

8. Hildebrands Jahresberichte über die Fortschritte der Chirurgie. I895I9I4.

9. Kappeler, Chirurgische Beobachtungen aus dem Kantonsspital Münsterlingen während der Jahre I865-I870.

10, Reverdin, Traitement chirurgical du goitre. Congr. fr. de chir. I908. Rev. de Chir. 
Über Störungen von seiten des Halssympathicus bei einfacher Struma usw. 423

II. Bossart, Über 1400 Kropfoperationen der Krankenanstalt Aarau. Festschrift Bircher Aarau.

I2. C. Brunner, Tumoren mit sekundären Nervenveränderungen. Korrbl. f. Schw. Ärzte i 9 Io. Nr. 15.

13. Monnier, Klinische Studie über die Strumektomie an der Hand von 670 Kropfoperationen. Bruns Beitr. I903. Bd. 54.

14. Steinegger, Über WundbehandIung, Wundverlauf und Wundfieber bei 400 Kropfoperationen. Beitr. z. klin. Chir.

15. Dufour, Compression exercée par le goitre. Apparition du syndrome de Horner. Rev. méd. de la Suisse romande. 1910.

16. v. Burg, Intrastrumöse Spontanblutungen. Beitr, z. klin. Chir. LXXVIII. 3. 